ARTÍCULO ORIGINAL

\title{
Problemas sanitarios de las majadas caprinas en los sistemas familiares de los valles calchaquies (Payogasta, Salta)
}

\author{
Suarez $\mathrm{VH}^{1^{*}}$, Martínez $\mathrm{GM}^{1}$, Olmos $\mathrm{LH}^{1}$, Arapa $\mathrm{C}^{2}$, Cortez $\mathrm{HS}^{1}$, Rojas $\mathrm{MC}^{3}$, Brihuega $\mathrm{BF}^{4}$, \\ Santillán $\mathrm{G}^{5}$, Álvarez $\mathrm{I}^{4}$, Goz $\mathrm{ML}^{6}$ \\ ${ }^{1}$ Estación Experimental Agropecuaria Salta (EEA Salta), Instituto Nacional de Tecnología Agropecuaria, \\ Argentina (INTA). \\ ${ }^{2}$ Municipalidad de Payogasta, Cachi, Salta, Argentina. \\ 3 Estación Experimental Agropecuaria Anguil "Ing. Agr. Guillermo Covas", INTA, Argentina. \\ ${ }^{4}$ Instituto de Investigación en Patobiología, Centro de Ivestigaciones en Ciencias Veterinarias y \\ Agronómicas, INTA, Buenos Aires, Argentina. \\ ${ }^{5}$ Departamento de Parasitología, Instituto Nacional de Enfermedades Infecciosas "Dr. Carlos G. \\ Malbrán", Buenos Aires, Argentina. \\ ${ }^{6}$ Laboratorio de Inmunoparasitología, Facultad de Ciencias Veterinarias, Universidad Nacional de La \\ Plata, La Plata, Argentina.
}

* Correspondencia: Víctor H. Suarez, EEA Salta, INTA, Ruta Nacional 68 km 172 (CP: 4403) Cerrillos, Salta, Argentina. E-mail: suarez.victor@inta.gob.ar

Recibido: 16 Abril 2020. Aceptado: 23 Julio 2020. Disponible en línea: 31 Julio 2020

Editor: P. Beldomenico

RESUMEN. Una encuesta transversal se llevó a cabo en 35 unidades productivas familiares (UPF) del Municipio de Payogasta (Salta) con el objetivo de describir los problemas sanitarios de las majadas. Se recabaron datos del manejo y sanidad de los animales. Se procesaron sueros caprinos para diagnóstico de brucelosis por BPA y FA, de artritis-encefalitis (CAEV) y clamidiosis por ELISA indirecto, toxoplasmosis y neosporosis por IFI, leptospirosis por microaglutinación. En heces caninas se diagnosticó echinococcosis por coproELISA. Se determinaron valores de $\mathrm{Cu}, \mathrm{Zn}, \mathrm{Mg}$ y Ca sérico por espectofotometría de absorción atómica. Se realizó recuento de huevos de helmintos en heces (hpg) y su diferenciación por coprocultivo. En el 80,6\% de las UPF se registró la ocurrencia de abortos y en el $73,3 \%$ la de ectima contagioso. El $84,8 \%$ de las UPF declararon problemas de mastitis y el $42,9 \%$ disturbios respiratorios graves en sus cabras. El porcentaje de UPF con seroprevalencia positiva a brucelosis fue de $2,9 \%$, leptospirosis de $20 \%$, clamidiosis de $66,7 \%$, toxoplasmosis de $76,9 \%$, neosporosis de $100 \%$ y CAEV de $26,7 \%$. Los promedios de los hpg fueron en junio, julio y octubre de 318,54 y 46 respectivamente, con Trichostrongylus y Haemonchus como nematodes predominantes. En el 51,4\% de las majadas se recuperaron huevos de Fasciola hepatica. El 41,6\% de las UPF tuvieron perros positivos a Echinococcus. Los promedios generales de cobre, zinc, magnesio y calcio séricos fueron respectivamente $0,78 \pm 0,13 \mathrm{ppm}, 0,63 \pm 0,23 \mathrm{ppm}, 1,96 \pm 0,25 \mathrm{mg} / \mathrm{d}$ y $12,2 \pm 0,9 \mathrm{mg} / \mathrm{dl}$. Estos resultados, además de generar los primeros antecedentes, muestran la importancia de profundizar los estudios para incrementar la producción caprina y el bienestar de las familias productoras.

SUMMARY. Disease problems of smallholder goat flocks in the Calchaquies Valleys (Payogasta, Salta). A cross-sectional survey was performed in 35 family farming units (FFU) in the Municipality of Payogasta (Salta), with the aim of describing disease presence in their goat flocks. Data on goat management and health were recorded. Sera were processed to diagnose brucellosis using the BPA and FPA test, to caprine arthritis-encephalitis (CAEV) and chlamydiosis by indirect ELISA, toxoplasmosis and neosporosis by IDIF and leptospirosis by microagglutination test. Dog echinococcosis was diagnosed by coproantigen ELISA test. Serum Cu, Zn, Mg and Ca were determined by atomic absorption spectrophotometer. Fecal samples were taken for eggs counts per gram of feaces (epg) and identifying genera by coproculture. Abortions were recorded in $80.6 \%$ of the FFU. Contagious echtyma was detected in $73.3 \%$ of the FFU. Mastitis and respiratory disorders were recorded in $84.8 \%$ and $42.9 \%$ of the FFU respectively. FFU positive seroprevalence of brucellosis was $2.9 \%$, leptospirosis $20 \%$, clamidiosis $66.7 \%$, toxoplasmosis $76.9 \%$, neosporosis $100 \%$ and CAEV $26.7 \%$. Mean epg were 318, 54 and 46 for June, July and October respectively, with Trichostrongylus and Haemonchus being the prevailing nematodes. Fasciola eggs were observed in $51.4 \%$ of the FFU. Positive dogs to Echinococcus were detected in $41.6 \%$ of the FFU. Mean serum cooper, zinc, magnesium and calcium per FFU were $0.78 \pm 0.13 \mathrm{ppm}, 0.63 \pm 0.23 \mathrm{ppm}, 1.96 \pm 0.25 \mathrm{mg} / \mathrm{d}$ y $12.2 \pm 0.9 \mathrm{mg} / \mathrm{dl}$, respectively. These results show the importance of making studies about the health of flocks in order to increase goat production and smallholder family welfare.

Palabras clave: encuesta, enfermedades caprinas, agricultura familiar, Valles Calchaquíes

Keywords: survey, goat, diseases, smallholder farming, Calchaquies Valleys 


\section{Introducción}

El clima y la orografía del municipio de Payogasta (Departamento de Cachi) se caracteriza por su extrema aridez, un relieve montañoso con los nevados de Cachi y de Palermo como marco y con una predominancia de serranías y quebradas por donde bajan los cursos de agua, los cuales son fundamentales para el riego de la producción agrícola y en menor medida para la cría del ganado menor. Estas actividades constituyen la principal fuente de ingresos de la población, compuesta casi en su totalidad por productores minifundistas propietarios de pequeños predios o en muchos casos arrendatarios $\mathrm{u}$ ocupantes de tierras fiscales. Los principales productos de estas economías familiares son el pimiento para pimentón y en menor medida alubias, tomate, comino, alfalfa, arvejas, maíz y la cría mayormente de cabras y en menor medida ovejas (Manzanal, 1987). Esta última actividad tiene como propósito la leche para la elaboración de quesos artesanales y la producción de cabritos, corderos y capones como principales productos tanto para autoconsumo o como para la venta de excedentes en las poblaciones cercanas como ocurre en otras regiones marginales de Argentina (Gutman et al, 2004; Martínez y Suarez, 2019).

Este tipo de cría extensiva de caprinos tiene graves condicionantes que afectan su competitividad y posibilidades de crecimiento en el Noroeste Argentino (NOA), como la escasa adopción de tecnologías simples e insumos en lo que hace al manejo alimenticio, reproductivo y sanitario de las majadas, como también la adopción de medidas y buenas prácticas que garanticen la inocuidad y calidad de los productos pecuarios (Suarez et al, 2017a; Martínez y Suarez, 2019).

En lo referente a los problemas sanitarios que limitan la producción caprina a lo largo del país existen escasas encuestas que describan las principales enfermedades y carencias minerales de los caprinos (Bedotti et al., 2002; Mancebo 2011). En la región del NOA desde hace algunos años se ha abordad esta problemática mediante el empleo de encuestas (Suarez et al, 2015; Suarez et al., 2017b), sin embargo la diversidad de ambientes y aspectos socioculturales de la región obliga a multiplicar esfuerzos debido a que las condiciones de explotación caprina varía de una ecorregión a otra. Cabe destacar que todavía falta información sobre el plano sanitario de los caprinos en algunas regiones y que aún restan estudios que ayuden a superar las limitantes sanitarias y posibiliten brindar la calidad necesaria a los productos pecuarios pensando en el bienestar de las familias criadoras y las posibilidades de ventas en el contexto de un turismo en crecimiento.

A partir de estos desafíos, el objetivo de la presente encuesta llevada a cabo entre los productores de caprinos del Municipio de Payogasta, fue caracterizar la presencia de enfermedades y carencias caprinas con el propósito de aportar información para abordar su estudio y así proponer y transferir tecnologías para mejorar la salud de las majadas en una región representativa de los Valles Calchaquíes de Salta.

\section{Materiales y Métodos}

\section{Tipo y lugar de la encuesta}

Una encuesta transversal se llevó a cabo entre junio y octubre del 2018 a partir de una visita a productores de 35 unidades productivas familiares (UPF) que criaban caprinos ubicadas en el Municipio de Payogasta, departamento de Cachi, provincia de Salta (Figura 1). Las UPF se ubicaron en los siguientes parajes: Buena Vista $(n=8)$, Payogasta $(n=7)$, Palermo $(n=6)$, Punta de Agua $(n=4)$, Tonco $(n=3)$, Belgrano $(n=3)$, Piul $(n=3)$, Arqualito $(n=1)$. El área de estudio está inserta en los denominados Valles Calchaquíes, región donde a sus habitantes los une una remota tradición cultural y una geografía que comparte montañas, valles y quebradas, de extrema aridez con precipitaciones promedio de alrededor de $200 \mathrm{~mm}$ ubicadas entre fin de diciembre y marzo.

La encuesta se realizó por conveniencia, seleccionando aquellas UPF representativas de la región de acuerdo a la tenencia de cabras según referentes del Municipio de Payogasta, que estaban dispuestas a recibir en su predio la visita de los encuestadores.

En cada visita la encuesta comprendió un cuestionario al productor, además de la toma de muestras en sus caprinos y la observación directa de eventuales patologías en la majada.

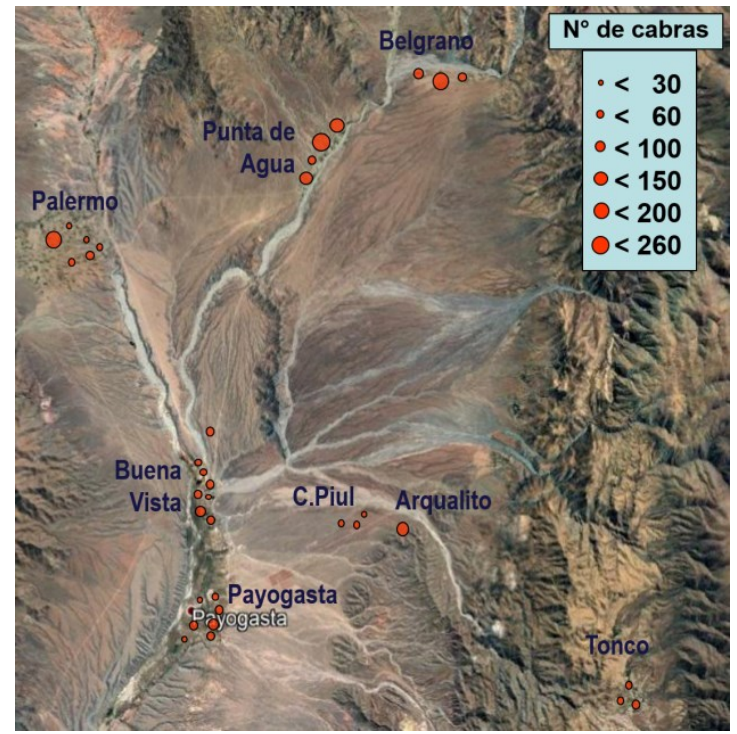

Figura 1. Ubicación de la zona del Municipio de Payogasta (2503'05,14" S; 66 06'03,37" 0; $2451 \mathrm{msnm})$ del Dto de Cachi, Salta, en donde se llevó a cabo la encuesta a las familias criadoras de caprinos. Se detallan los parajes en donde se llevó a cabo la encuesta y el tamaño de las majadas caprinas. 


\section{Cuestionario}

A partir de la respuesta de los productores se completó una encuesta, que en su parte inicial se recabaron datos generales y de manejo (Suarez et al., 2020), mientras que en su parte principal datos de los problemas sanitarios de la majada referidos, al número y categoría de caprinos que padecieron problemas de salud durante el último año. Solo se registraron tanto aquellas patologías fácilmente reconocibles a partir de la descripción del productor, como aquellas con diagnóstico previo veterinario o de laboratorio, más aquellas observadas directamente durante la visita. Con tal fin, la encuesta se complementaba para guía de los productores con fotografías de rumiantes menores o de sus órganos con diferentes patologías o parasitosis. Para aquellos casos sanitarios confirmados a partir del relato de los productores, se registró la morbilidad, mortalidad, categoría, época del año de ocurrencia y tratamiento.

\section{Revisación clínica, toma y análisis de muestras}

En cada una de las UPF se inspeccionaron clínicamente un total de 20 cabras junto al total de los machos reproductores (aproximadamente 2 por UPF), determinando edad por revisación dentaria, problemas mamarios, podales, oculares, de piel, del aparato reproductor externo, secuelas de ectima contagioso, presentación de diarreas y disturbios respiratorios, parásitos externos, etc.

Se tomaron en promedio por UPF 21,3 muestras (máx= 25 y $\min =15$ ) de sangre mediante punción yugular y de materia fecal. Se procesaron sueros para el diagnóstico de brucelosis por la prueba tamiz de antígeno bufferado en placa (BPA) y confirmadas por polarización fluorescente (FPA). También se realizaron las serologías para el diagnóstico de presencia de anticuerpos contra artritis encefalitis caprina (CAEV) y clamidiosis, por ensayo inmunoenzimático indirecto (I ELISA). Se utilizó la técnica de aglutinación microscópica con antígenos vivos para serodiagnóstico de leptospirosis y la prueba de inmunofluorescencia indirecta para el diagnóstico de toxoplasmosis y neosporosis. Además, se recolectaron 33 muestras de heces de perros de las UPF para ser analizadas por la técnica de coproELISA para detectar antígenos secretorio/excretorios de Echinococcus. granulosus.

A partir del suero se determinaron los valores de cobre, zinc, magnesio y calcio por espectrofotometría de absorción atómica de llama (Perkin Elmer AAnalyst, 200).

En heces se realizó el recuento de huevos de helmintos y ooquistes por gramo de materia fecal (hpg) y la diferenciación de géneros por coprocultivo, además de la técnica de Baermann para recuperar nematodes pulmonares (Suárez, 1997). Se utilizó el método de sedimentación y coloración de azul de metileno para recuperar huevos de Fasciola hepatica (Viñabal et al., 2015).

\section{Análisis de los datos}

Los datos fueron chequeados a diferentes niveles como veterinarios locales, extensionistas del INTA, agentes sanitarios departamentales para descartar errores y en algunos casos de inconsistencias se volvió a llamar telefónicamente al productor a través del municipio. Para el análisis estadístico de las variables se utilizaron técnicas descriptivas y medidas de resumen como el número de observaciones, media aritmética y desvío estándar (Thrusfield, 1997).

\section{Resultados y discusión}

\section{Características de las UPF y prácticas de manejo}

Las UPF muestreadas criaban en promedio $80,1 \pm 57,1$ caprinos, sin contar los cabritos menores a los 45 días de edad $(44,7 \pm 41,6)$ debido a que su número al momento de las visitas variaba de acuerdo si estaban en plena parición o si habían sido consumidos o vendidos. Aunque las características productivas ya fueron analizadas previamente (Suarez et al., 2020) se detallarán brevemente algunas características del manejo llevado a cabo por los productores de las UPF. La composición genética de las majadas era en más del $60 \%$ de cruzamientos Anglo Nubian con biotipos de raza Criolla. En cuanto a la cría de otras especies el $82,4 \%$ de las UPF criaba ovinos $(12,2 \pm 15,8)$ y el $20,6 \%$ un escaso número de bovinos $(2,7 \pm 9,6)$. El sistema productivo identificado resultó de tipo extensivo con encierre nocturno y la alimentación basada en el pastoreo de los cerros, de alfalfa, rastrojos o residuos de cosecha. El $100 \%$ de las UPF practicaba el ordeñe manual en los corrales. En el $51,4 \%$ de las UPF el servicio era estacionado, mientras que en el resto continuo. Las instalaciones de las UPF eran precarias, contando solamente con uno a dos corrales y pocos de ellos con algo de reparo. El recurso agua de bebida para la majada era en general suficiente y de buena calidad.

En cuanto a las prácticas sanitarias ningún productor declaró usar vacunas en su majada y solo el 8,6 \% inyectaba complejos minerales-vitamínicos una o dos veces al año a toda la majada en forma rutinaria. El 65,7\% de los productores desparasitaba contra nematodes gastrointestinales y Fasciola hepatica a la totalidad de la majada, mayormente en otoño $(52,9 \%)$ o primavera $(41,2 \%)$ con ivermectina, closantel y o albendazole.

\section{Tasas de mortalidad y abortos}

El promedio de la tasa de mortalidad anual por majada fue del $16,1 \pm 7,5 \%$ sin contar las muertes perinatales. La mortalidad de animales adultos promedio fue del $10,2 \pm 7,4 \%$, y la del destete y de la reposición respectivamente fue del $10,6 \pm 7,5 \%$ y $8,3 \pm 11,1 \%$. La estimación de los datos referidos a la tasa de mortalidad perinatal a partir del relato de los productores muestra un promedio general del 14,9 $\pm 9,2 \%$, valor que se ve incrementado en las UPF que no estacionan 
servicio (Suarez et al., datos no publicados). Estos valores son altos, aunque están atenuados por la presencia de más de una parición anual de algunas cabras en estos sistemas. Entre las causas de muertes de los cabritos antes de las $48 \mathrm{~h}$ de vida, al igual a lo declarado en otras regiones (Suarez et al., 2016), los dueños las adjudicaron principalmente al frío y el viento y en menor medida a aquellos los partos que coincidían con lluvia y tormentas. Debido a las características de este estudio no fue posible precisar las causas de las muertes ocurridas antes de las $48 \mathrm{~h}$ de vida de los cabritos. En cuanto a las muertes de cabritos por predación (pumas, zorros), esta fue considerada como de poca importancia ya que la mayoría de los partos ocurre en los corrales permaneciendo los cabritos allí y solo aquellas cabras que parían anticipadamente en los cerros estaban expuestas a los predadores.

En cuanto a la ocurrencia de abortos en el $80,6 \%$ de las UPF declararon su presencia y de estas un $65,5 \%$ mencionó que las cabras padecían retención de placenta. El promedio intramajada de abortos comunicados fue del $8,1 \pm 7,1 \%$ de las hembras en parición con extremos que oscilaron entre el 1,6 y el $28,0 \%$. Se comunican tanto abortos de fetos grandes como de chicos. Los promedios coinciden con lo comunicado en una encuesta previa realizada en las quebradas áridas de Salta y Jujuy donde la tasa de abortos fue del 8,6 \pm $8,5 \%$ (Suarez et al., 2016).

\section{Enfermedades infecciosas}

En algunas de las UPF se observaron costras y pápulas sugerentes de la presencia de ectima contagioso, tanto en las mucosas de cabritos como en los pezones de las cabras. Con la ayuda de las ilustraciones la presencia de esta enfermedad fue declarada en un $77,3 \%$ de las UPF, aunque en la mayoría de ellas los productores la señalaron como de poca importancia. Solo en un $36 \%$ de esas UPF, los propietarios declararon la ocurrencia de brotes importantes aproximadamente cada 3 años, sobre todo en la parición de otoño cuando los animales descienden de los cerros. Su presencia en las majadas coincide con otra encuesta realizada en la Quebradas de Humahuaca y del Toro donde en un $75 \%$ de las UPF la reconocieron (Suarez et al., 2016).

El $84,8 \%$ de las UPF se registraron problemas de mastitis, fundamentalmente durante el verano, las cuales fueron descriptas con la aparición de leche sanguinolenta o cremosa o hinchazón y pérdida del medio mamario y a veces muerte. Se estimó a partir del relato de las encargadas del ordeñe y de la observación directa una prevalencia del $8,4 \pm 0,6 \%$ y una mortalidad del $1,2 \pm 0,2 \%$. Las descripciones de los casos de muerte por mastitis al igual a las obtenidas en otras encuestas (Suarez et al, 2016; 2017b) se podrían asociar a mastitis gangrenosas previamente observadas en tambos comerciales debidas a Staphylococcus aureus (Suarez et al., 2014). La mayor incidencia se da durante el verano que es el período donde ocurren las precipitaciones y probablemente se deba a la acumulación de barro en los corrales, la suciedad de las ubres y a una mayor tasa de trasmisión y proliferación bacteriana.

Un 39\% de los propietarios declararon casos de muertes agudas, pero sin poder confirmar un diagnóstico preciso pudiéndose tratar de intoxicaciones o de alguna enfermedad clostridial, ya que en ninguna UPF se vacuna contra enfermedades clostridiales.

En un $24 \%$ de las UPF se reportaron problemas respiratorios graves en cabritos lactantes que nunca engordan, con moco, tos, decaimiento, ahogo y a veces muerte en pocos días y a pesar de la terapia antibiótica. En las cabrillonas y cabras se declararon disturbios respiratorios graves (tos, jadeo, temblores y fiebre) mayormente en invierno en un $42,9 \%$ de las UPF, que en algunos casos afectan hasta el $50 \%$ de los animales. No fue posible contar con un diagnóstico preciso de los disturbios respiratorios relatados.

Se declararon casos de diarreas en el $45,2 \%$ de las UPF, pero como eventos no muy graves y de baja prevalencia. Fueron descriptos en cabritos menores al mes de vida cursando con heces blandas amarillentas y cólicos abdominales como también descripciones ligadas a disturbios en la alimentación como excesos de leche ("empacho"). Se sabe que los trastornos digestivos pueden obedecer a causas no infecciosas como indigestiones por cambios bruscos en la ingesta o por causas climáticas como el frío por falta de reparo suficiente, como también a causas infecciosas por asociación de virus, bacterias y/o parásitos. En condiciones similares existen diagnósticos, previos de colibacilosis, coccidiosis $y$ trichuriasis en cabritos menores de tres meses de edad (Bedotti y Sánchez Rodríguez, 2002; Suarez et al., 2013).

En un $85,7 \%$ de las UPF se observaron animales con abscesos submandibulares o en la parte del cuello, (chupones, apostemas) que segregaban pus verdoso, típicos de linfoadenitis caseosa (Corynebacterium pseudotuberculosis). Según el relato, se estimó que un $3,9 \pm 6,2 \%$ de los animales presentaba abscesos, pero que solo un $20 \%$ de los propietarios dio importancia al problema debido bajo número de cabras afectadas.

Solo en un $20 \%$ de las UPF los propietarios declararon tener cabras con afecciones podales ("uñeras") durante los períodos de lluvias en verano, pero restándole importancia debido a una ocurrencia muy baja. A diferencia de otras regiones con un régimen de precipitaciones más elevadas como en el chaco salteño donde la ocurrencia es mayor (Suarez et al., 2015), en la presente región la aridez y las pendientes que impiden la acumulación de barro en los corrales serían las causales de esta menor prevalencia.

\section{Serodiagnóstico de enfermedades}

En la Tabla 1 se presentan los agentes etiológicos estudiados y su prevalencia a nivel de majadas en las 
UPF encuestadas y a nivel existencias en la región estudiada.

En brucelosis, a excepción de solo un suero que fue dudoso, todas las muestras fueron negativas confirmando una tendencia que se venía registrando en las quebradas áridas y valles templados donde las encuestas fueron siempre negativas (Gaido et al., 2010, Suarez et al., 2017) y alerta sobre la importancia de controlar mediante el diagnóstico de brucelosis aquellos animales que fueron adquiridos en otras regiones. Una región libre de brucelosis posee una ventaja competitiva muy importante al momento de ofrecer sus productos pecuarios.

Otras regiones no ofrecen estas ventajas ya que, en San Juan, Mendoza, Formosa o el chaco salteño las encuestas resultaron con un $25,4 \%, 28,1 \%, 29,8 \%, 17,9 \%$ de majadas caprinas positivas respectivamente (COPROSA San Juan 2007; Robles et al., 2007; Mancebo et al., 2011; Gaido et al., 2011).

El porcentaje de UPF donde se hallaron reactores positivos a leptospirosis fue del $20 \%$ con tasas de prevalencia intramajada cercanas al 33\%, con bajos títulos que no superaron 1:200 y con solo la presencia del serovar $L$. grippotyphosa. Estos resultados indican la presencia de Leptospira pero con una prevalencia moderada que coincidió con la de otras encuestas (25\%) realizadas a nivel de las quebradas áridas de Jujuy y Salta (Suarez et al., 2016) y se diferenció de lo hallado en el departamento de Rivadavia en el este salteño donde hubo prevalencia a nivel UPF del 70\% y títulos de hasta 1:800 del serovar $L$. pomona (Suarez et al., 2015).

En un $76,9 \%$ de las UPF se hallaron caprinos positivos a toxoplasmosis (Toxoplasma gondii) con una prevalencia general cercana al $48,2 \%$ y una prevalencia intramajada que osciló entre el 20 al $80 \%$. La tasa de UPF con presencia de caprinos seropositivos hallados coinciden con los resultados para la provincia de Salta de Dodero et al. (2019), donde un $79,4 \%$ de las UPF tienen animales seropositivos pero con una menor prevalencia $(14,8 \%)$ general. Otras encuestas llevadas a cabo en La Pampa $(2,6 \%)$, San Luis $(19,8 \%)$, Córdoba (33\%) y Buenos Aires (63\%) mostraron respectivamente disímiles niveles de cabras seropositivas (Bedotti y Sánchez Rodríguez, 2002; Gos et al., 2014; 2017).

Estas altas tasas de prevalencia tienen sentido debido a que la transmisión de la toxoplasmosis no solo se genera por el contacto con las heces de los felinos (infección horizontal a través del consumo de agua o forrajes contaminados con ooquistes), sino también por transmisión vertical a través de las cabras preñadas e infectadas al feto o a través de la leche al cabrito (Dubey, 2010). Además, la alta seroprevalencia atenta contra la producción y sobre todo alerta sobre la necesidad de llevar adelante medidas de prevención que alerten y garanticen la salud de las familias productoras que están en estrecho contacto con las cabras, advirtiendo del peligro de contraer toxoplasmosis por consumir carne mal cocida, leche cruda o agua contaminadas.

En el $100 \%$ de las UPF se diagnosticaron cabras con anticuerpos contra Neospora caninum con prevalencias intra-majada que oscilaron entre el 20 y el $100 \%$ de los animales sangrados. Estos hallazgos, más el reciente reporte en el país sobre la transmisión de madre caprina a feto (Campero et al., 2018), ameritan estudios sobre qué importancia sobre los abortos y natimortos tendrían las infecciones con $N$. caninum. A pesar de que existen todavía interrogantes sobre la importancia de la neosporosis en las cabras y ovejas, los estudios actuales muestran grandes similitudes con el bovino, ya que sería una enfermedad más importante sobre la reproducción caprina de lo que se creía (Porto et al., 2016).

En un $66,7 \%$ de las UPF se hallaron caprinos seropositivos a clamidiosis o aborto enzoótico de los pequeños rumiantes, causado por Chlamydia abortus. Aunque las muestras no fueron numerosas, su prevalencia que

Tabla 1. Resultados de los serodiagnósticos frente a las diferentes enfermedades. Número de unidades productivas familiares (UPF) estudiadas, número y porcentaje de UPF positivas, número de caprinos sangrados y número y porcentaje de positivos. *Referido a un suero de diagnóstico dudoso.

\begin{tabular}{|c|c|c|c|c|c|c|}
\hline Enfermedades & $\mathbf{N}^{\circ}$ de UPF & $\begin{array}{l}\mathrm{N}^{\circ} \text { de UPF } \\
\text { positivas }\end{array}$ & $\begin{array}{l}\% \text { de UPF } \\
\text { positivas }\end{array}$ & $\begin{array}{l}\mathrm{N}^{\circ} \text { de caprinos } \\
\text { sangrados }\end{array}$ & $\begin{array}{l}\mathbf{N}^{\circ} \text { de caprinos } \\
\text { positivos }\end{array}$ & $\begin{array}{l}\text { Porcentaje de } \\
\text { sero-positivos }\end{array}$ \\
\hline Brucelosis & 35 & 1 & $2,9 *$ & 751 & $1^{*}$ & $0,13^{*}$ \\
\hline Leptospirosis & 15 & 3 & 20 & 139 & 10 & 7,2 \\
\hline Toxoplasmosis & 13 & 10 & 76,9 & 141 & 68 & 48,2 \\
\hline Artritis-encephalitis caprina & 15 & 4 & 26,7 & 120 & 6 & 5,0 \\
\hline Clamidiosis & 9 & 6 & 66,7 & 58 & 6 & 10,3 \\
\hline
\end{tabular}


osciló entre 11,1 y $33,3 \%$ es un llamado de atención. También en una encuesta previa en las quebradas áridas de Jujuy y Salta hubo caprinos seropositivos en el $88,8 \%$ de las majadas (Suarez et al., 2016). A nivel país sólo hay escasas evidencias serológicas en caprinos (Fiorentino et al, 2015) y un caso diagnosticado en el oeste de la provincia de La Pampa (Bedotti et al., 2008). Como se trata de una enfermedad que a nivel mundial es responsable de pérdidas productivas, mayormente abortos al final de la gestación y una zoonosis especialmente de riesgo en las mujeres embarazadas, sería relevante encarar futuras investigaciones sobre la real importancia de la presencia de Chlamydia spp. en la agricultura familiar del NOA.

Se hallaron un $26,7 \%$ de UPF con reactores positivos al virus de la artritis encefalitis caprina (CAEV), aproximándose a los resultados con un relevamiento a nivel nacional donde se hallaron mayormente en el este de Salta un $30 \%$ de predios positivos y un $7,3 \%$ de cabras positivas (Trezeguet et al., 2010). Sin embargo, estos datos contrastan con datos más recientes de la provincia de Salta donde solo se hallaron 6,25\% de majadas positivas, pero en ese mismo trabajo se describe un caso clínico de CAEV, donde hubo un 55\% de cabras seropositivas y un $48 \%$ con signos clínicos (Dodero et al., 2017). Este último caso, sumado a otros eventos descriptos en el oeste pampeano (Bedotti et al., 2007), San Luis (Rossanigo et al., 2016) y el aislamiento de una cepa de caprinos de la Buenos Aires (Panei et al., 2017), confirman la circulación de la enfermedad en el país.

\section{Presencia de parásitos y enfermedades parasitarias}

Los promedios de los conteos de huevos de nematodes gastrointestinales NGI, de Fasciola por gramo de heces y los porcentajes de géneros de nematodes diferenciados de acuerdo a los períodos de muestreo se presentan en la Tabla 2. Trichostrongylus seguido por Haemonchus y en menor medida Teladorsagia fueron los géneros de nematodes prevalentes. Esporádicamente se observaron huevos de los géneros Oesophagostomum y Trichuris. Coincidiendo con la presencia observada de Haemonchus spp., el $28 \%$ de los productores declaró haber visto en el abomaso a la faena al gusano de cuajo.

Los hpg observados fueron en general bajos, decreciendo desde finales de otoño hacia la primavera. A partir de estos resultados se podría inferir que la mayor infestación se registraría al inicio del otoño cuando el desarrollo de los NGI se vería favorecido por las lluvias estivales. A pesar de que los muestreos de heces no mostraron los niveles de infestación descritos en las explotaciones comerciales de los valles templados de altura de Salta y Jujuy (Suarez et al., 2017c), las principales especies presentes coincidieron con las de esa región. En otros muestreos en otras regiones áridas también se observó la presencia en bajo número de los géneros Trichostrongylus spp. y de Haemonchus spp. (Suarez et al., 2018)

El $50 \%$ de los productores reconocieron la presencia de Fasciola hepatica ("unca") en sus majadas y de los inconvenientes que ocasionaban en la salud de sus animales. Esto fue corroborado a partir de los conteos de huevos de Fasciola que dieron positivas en el 51,4\% de las majadas (Tabla 2). Otros muestreos en las Quebradas del Toro y de Humahuaca mostraron la importancia de este trematode en la producción de las majadas (Suarez et al., 2016)

Tabla 2. Promedio general de los hpg, conteos de Fasciola por $\mathrm{g}$ de heces y porcentajes de géneros de nematodes gastrointestinales recuperados de los coprocultivos durante cada muestreo del estudio.

\begin{tabular}{|c|c|c|c|}
\hline & 6 -jun & 18 -jul & 24-oct \\
\hline hpg & 318,2 & 54,3 & 46 \\
\hline Haemonchus & 17,3 & 3,6 & 69,5 \\
\hline Trichostrongylus & 65,2 & 74 & 18 \\
\hline Teladorsagia & 17,5 & 21,3 & 5 \\
\hline Oesophagostomum & 0 & 1 & 7 \\
\hline Trichuris & 0 & 0 & 0,5 \\
\hline Fasciola por g & 0,8 & 4,5 & 10,5 \\
\hline
\end{tabular}

Tabla 3. Promedio y desvío estándar de los valores séricos de cobre, zinc, magnesio y calcio de las cabras de las unidades productivas encuestadas.

\begin{tabular}{|l|c|c|c|}
\hline Minerales & N & promedio & DS \\
\hline Cobre ppm & 286 & 0,78 & 0,13 \\
\hline Zinc ppm & 286 & 0,63 & 0,23 \\
\hline Magnesio mg/dl & 286 & 1,96 & 0,25 \\
\hline Calcio mg/dl & 286 & 12,29 & 0,92 \\
\hline
\end{tabular}

En un 21\% de las UPF los propietarios describieron casos de animales con signos nerviosos (marcha en círculos, como ciegos), los cuales podrían tratarse de signos de cenurosis. Diagnósticos previos muestran la presencia de Coenurus cerebralis en Salta (Cafrune et al., 2014).

En el $54,3 \%$ de las UPF se describió la presencia a la faena de quistes en hígados y pulmones compatibles con hidatidosis de acuerdo a las fotográficas presentadas, lo que fue corroborado a partir del estudio de la presencia de copro-antígenos de Echinococcus. granulosus en las heces de los perros de los propietarios donde un $41,6 \%$ tuvieron animales positivos. La prevalencia general fue del $45,5 \%$ de perros positivos. En todas las UPF había perros, cuyo promedio fue de $4 \pm 2,3$ con extremos de 1 a 12 canes. De acuerdo a la información obtenida, las intervenciones de tipo profiláctico basadas en la desparasitación de perros 
fueron esporádicas y de acuerdo a estos resultados sin el éxito esperado. Sería de suma importancia a la luz de estos datos que organismos oficiales puedan hacer una encuesta a nivel regional y provincial para evaluar la necesidad o no de utilizar la vacuna EG95 contra la hidatidosis.

En cuanto a ectoparásitos sólo se observaron piojos chupadores (Linognathus stenopsis) en un $67,7 \%$ de las UPF, de las cuales un $38,1 \%$ de los propietarios los consideraban como un factor negativo a tener en cuenta para el desarrollo de los cabritos. La mayor densidad de piojos era vista en invierno e inicios de primavera, siendo objeto de tratamientos frecuentes con insecticidas aplicados por derrame dorsal o inyectables (avermectinas).

Un $38,7 \%$ de propietarios informaron haber tenido casos de sarna (Psoroptes ovis) en las ovejas. En una UPF se dijo haber visto sarna en las orejas de las cabras, por lo que se podría tratar de sarna psoróptica. En los ovinos se observó la presencia del díptero melófago (Melophagus ovinus).

Las miasis (Cochliomya hominivorax) según las declaraciones de la mayoría de los propietarios, revisten poca importancia, ocurriendo a partir de noviembre-diciembre, mientras que los disturbios respiratorios con importante producción de moco declarados (en un $85,7 \%$ de UPF) y principalmente vistos desde el final del invierno y durante el verano también fueron tenidos como problema menor. En algunos casos estos signos clínicos pudieron asociarse al gusano del cuerno (Oestrus ovis).

Problemas carenciales y tóxicos:

En un $18,5 \%$ de las UPF se registró hipotiroidismo, a partir de la observación de bocio mayormente en caprinos jóvenes. La mayoría de los propietarios declararon tener 2 a 3 animales con bocio ("coto") por majada, pero que antes se observaban más casos de bocio que en la actualidad. Probablemente el uso generalizado de sales para lamer redujo el número de animales con esta manifestación clínica.

En otras encuestas caprinas como en el oeste de Formosa o en los valles templados del NOA se registró una mayor prevalencia de bocio respectivamente en un $27,6 \%$ o en un $58 \%$ de los predios (Mancebo et. al., 2011, Suarez et al, 2017).

Los valores de cobre sérico hallados estuvieron en promedio dentro de valores considerados normales (tabla 3 ) con solo en un $18,2 \%$ de las UPF con registros de valores marginales (de 0,53 a 0,65 ppm). Coincidentemente, un $18,6 \%$ de los productores describieron signos que podrían interpretarse como de ataxia enzoótica: cabritos débiles, como deslomados y muertos antes de la primera semana de vida. En otras regiones los valores de cobre sérico hallados fueron más bajos y marginales, como en las quebradas áridas del Toro y de Humahuaca $(0,48 \pm 0,10 \mathrm{ppm}$; Suarez et al., 2016), en el Chaco salteño $(0,49 \pm 0,12 \mathrm{ppm}$; Suarez et. al., 2015) o en San Luis $(0,42 \pm 0,20$ ppm; Sager y Rossanigo, 2002).

Los valores de zinc sérico hallados estuvieron en promedio también dentro de valores considerados normales (tabla 3 ), aunque en un $40,9 \%$ de las UPF se registraron majadas con valores marginales (de 0,37 a $0,48 \mathrm{ppm})$. En otras encuestas en el NOA los valores registrados para este mineral estuvieron dentro de los rangos de normalidad (Suarez et al., 2015; 2016; 2017).

El promedio general de los muestreos relativos al magnesio y calcio séricos fueron normales como se presenta en la tabla 3, coincidiendo con las encuestas caprinas de otras regiones de Salta (Suarez et al., 2015; 2017), aunque diferenciándose de otras en las quebradas áridas donde los valores de calcio fueron bajos (7,25 $\pm 2,25 \mathrm{mg} / \mathrm{dl}$, Suarez et al., 2016).

Un $31,8 \%$ de los propietarios declararon tener problemas de desnutrición y muertes durante el período inverno primaveral, coincidiendo con lo que acontece en otras regiones y sistemas productivos del NOA (Martínez y Suarez, 2019).

En cuanto a problemas metabólicos, en un $66,7 \%$ de las UPF se registraron casos de timpanismo y muertes debido al empaste e hinchazón en el pastoreo de alfalfa, donde el $35 \%$ de los productores declararon pérdidas importantes de entre el 5 y el $10 \%$ de sus cabras.

Entre las enfermedades atribuidas posiblemente a causas tóxicas, sobresalió el relato de algunos propietarios $(21,1 \%)$ referente a muertes de animales en los ciénagos (áreas pantanosas) circundantes al río Calchaquí, ocurridas cuando las aguas de se tornan oscuras durante el período de lluvias. Los signos clínicos descriptos en estos casos fueron problemas para respirar (disnea), ahogo, cuello estirado, envaramiento e incoordinación con líquido gelatinoso o sangre barrosa en la cavidad intestinal. Este cuadro de insuficiencia respiratoria podría relacionarse con una intoxicación por nitritos a partir del consumo de plantas o agua rica en nitratos que podría tener como fuente los productos de la fertilización y el guano provenientes de las parcelas agrícolas.

Entre otras intoxicaciones debidas a plantas solo en dos UPF se refirieron a pocas muertes de animales jóvenes, sin experiencia como cabrillonas. consumiendo hediondilla o duraznillo negro (Cestrum parqui).

\section{Otros problemas:}

En cuanto a la importancia de la predación para las cabras, en el $41,9 \%$ de las UPF se adujo tener problemas. Aquellos productores ubicados en los parajes más alejados declararon como los principales predadores a los pumas y secundariamente a los zorros 
y aquellos cercanos al poblado de Payogasta a los perros domésticos.

Conclusiones:

Los presentes resultados, con todas sus limitantes de diseño en cuanto al número y a la elección de las UPF, muestran cuales podrían ser las principales limitantes sanitarias de la cría caprina asociada a los sistemas de la agricultura familiar de la región estudiada.

En cuanto a las enfermedades que afectan la productividad de las majadas y deberían ser estudiadas en profundidad podemos destacar dentro de las infecciosas a las mastitis y en un segundo plano a los disturbios respiratorios y dentro de las parasitosis la presencia de Fasciola hepatica. Cabe destacar, que solo una cabra fue positiva a brucelosis, y con poco esfuerzo sería posible de erradicar y así potenciar los productos regionales.

El nivel en las majadas de abortos y sus consecuencias productivas como en lo que hace a la salud de las familias productoras debe ser también un tema de estudio prioritario sobre todo aquellas zoonosis como toxoplasmosis y clamidiosis o aquellas que afectarían la reproducción como la neosporosis.

La alta presencia de echinococcosis hallada en las UPF amerita su investigación y la toma de medidas de prevención de esta zoonosis en la región.

Finalmente, a pesar de que el muestreo solo abarcó el municipio de Payogasta, de acuerdo a las similitudes halladas en otras regiones de valles y quebradas áridas del NOA, estos resultados posibilitarían su extrapolación a otras zonas de los valles Calchaquíes y servir de sustento $y$ antecedentes para futuras investigaciones sobre cómo controlar las enfermedades y problemas sanitarios o zoonosis más relevantes.

\section{Agradecimientos}

Los autores quieren expresar su agradecimiento a las familias por su gran predisposición y participación en la encuesta a partir de sus opiniones. También un reconocimiento al Municipio de Payogasta por contribuir con el financiamiento y con la ejecución del presente trabajo.

\section{Bibliografía}

Bedotti DO, Sánchez Rodríguez M. 2002. Observaciones sobre la problemática sanitaria del ganado caprino en el oeste Pampeano. Vet. Arg. 19(182): 100-112.

Bedotti DO, Fort MC, Giménez $\mathrm{H}$, Langhoff A, Garré J, Hertsommer O. 2007. Descripción de un caso de
Artritis-Encefalitis caprina en la provincia de La Pampa, Argentina. V Congreso de Especialistas en pequeños rumiantes y camélidos sudamericanos, Mendoza, Argentina. Mayo 2007. pp: 163-165.

Bedotti DO, Fort MC, Fuchs L, Giménez H, Urquiza J. 2008. Descripción de un caso de aborto por Chlamidya psitacci en un establecimiento caprino ubicado en el departamento Puelen, Provincia de La Pampa. Resúmenes XVII Reunión Científica Técnica de la AAVLD, Santa Fe. Octubre 2008, p. B28.

Cafrune MM, Grossberger G, Viñabal AE, Aguirre DH. 2014. Caso de cenurosis (Coenurus cerebralis) clínica crónica en ovinos de Salta, Argentina. 20a Reun. Anu. Asoc. Arg. Vet. Lab. Diag., S.M. de Tucumán, Noviembre 2014, 1 p.

Campero LM, Gos ML, Moore DP, Regidor Cerrillo JR, Unzaga JM, Moré GA, Ortega Mora LM, Venturini MC. 2018. Microsatellite pattern analysis of Neospora caninum from a naturally infected goat fetus. Vet. Parasitol. 255: 58-60.

Dodero AM, Micheloud JF, Alfaro JR, Alfaro EJ, Pinto GB, Suarez VH. 2017. Caracterización de la enfermedad de la artritis y encefalitis caprina en las provincias de Salta y Jujuy. FAVE Sección Ciencias Veterinarias 16: 7-12.

Dodero AM, Bertoni AE, Cortez HS, Salatin AO, Martínez Almudévar F, Gos ML, Suarez VH. 2019. Toxoplasmosis caprina en la provincia de Salta. FAVE Sección Ciencias Veterinarias 18: 1-5.

Dubey JP. 2010.Toxoplasmosis of animal and humans. 2nd Edition. CRC Press. Boca Raton. FL, USA. 336 pp.

Fiorentino MA, Brunello GE, Castro MA, Cabral Ortiz DA, Aguilera N, Villagran E, Vera TA. 2015. Serología positiva a Chlamydia abortus en cabras con antecedentes de abortos del Dto de Chamical, provincia de La Rioja. Resúmenes 9no Seminario de la Fundación Charles Louis Davis en Argentina. Salta, Septiembre 2015.

COPROSA San Juan. 2007. Programa de control de la Brucelosis caprina. Provincia de San Juan. Propuesta 2007/2016: 14 pp.

Gaido $A B$, Colque Puppi F, Bravo RD, Vorano AV, Salatin AO, Aguirre DH. 2010. Brucelosis caprina: Encuesta serológica en majadas de tres departamentos de los Valles Calchaquíes, provincia de Salta. XVIII Reunión Científico Técnica Asoc. Arg. Vet. Lab. Diag., Mercedes, Corrientes. p.109. 
Gaido AB, Salatin A, Neumann RD, Marinconz $R$, Rossetto C, Aguirre N, Suárez VH, Aguirre DH. 2011. Goat brucellosis: a serological study in flocks from the east of Salta, Argentina. Brucellosis 2011 International Research Conference. Buenos Aires, p. 94.

Gos ML, Delgado MG, Bonzo EB, Arnonaga C, Pardini L, Unzaga JM, Rodriguez $M$, Moré GA, Venturini MC. 2014. Presencia de anticuerpos para Toxoplasma gondii y Neospora caninum en caprinos del departamento de Belgrano, provincia de San Luis, Argentina. XX Reunión Científico Técnica de la Asociación Argentina de Veterinarios de Laboratorio de Diagnóstico, Tucumán, Argentina.

Gos ML, Manazza JA, Späth EJA, Pardini L, Fiorentino MA, Unzaga JM, Moré GA, Venturini MC. 2014 Seroprevalence of Toxoplasma gondii and Neospora caninum infections in goats from two Argentinean provinces. Open Vet. J. 7: 319-322

Gutman G, Iturregui ME, Filadoro A. 2004. Propuestas para la formulación de políticas para el desarrollo de tramas productivas regionales: El caso de la lechería caprina en Argentina. Informe CEPAL, 94 pp.

Mancebo OA, Russo AM, Giménez JN, Gait JJ, Monzón CM. 2011. Enfermedades más frecuentes en caprinos de la provincia de Formosa (Argentina). Veterinaria Argentina 28 (274): 1-16.

Manzanal M. 1987. Pobreza y marginalidad en el agro argentino. La producción agrícola y su comercialización en Cachi, Salta. Cuadernos del CEUR, Buenos Aires, Argentina. ISSN 0326-1417.

Martínez GM y Suarez VH. 2019. Lechería Caprina: producción, manejo, sanidad, calidad de leche y productos. 1ra Ed. INTA Ediciones, Colección Investigación, desarrollo e innovación. 167 pp.

Panei JC, Gos ML, Valera AL, Galosi CM, Echeverria MG. 2017. First isolation and nucleotide comparison of the gag gene of the caprine arthritis encephalitis virus circulating in naturally infected goats from Argentina. Open Vet. J. 7: 32-35.

Porto WJN, Regidor-Cerrillo J, de Cássia Peixoto Kim P Benavides J, dos Santos AC, Horcajo SP, da Fonseca Oliveira AA, Ferre I, Mota RA, Ortega-Mora Porto LM 2016. Experimental caprine neosporosis: the influence of gestational stage on the outcome of infection. Vet. Res. 47: 29

RIAN. 2010. Existencias ganaderas caprinas 2009-
2010. Red de Información Agropecuaria Nacional, INTA RIAN, rian.inta.gov.ar/ [fecha de consulta: 14 diciembre 2019].

Robles C, Bernard O, Zenocrati L, Marcellino R. 2007. Encuesta serológica sobre Brucelosis en caprinos de la provincia de Mendoza. Vet. Arg. 24(233): 172-185.

Rossanigo C, Delgado C, Carosio A, Raia A, Alvares I, Rodriguez M, Paje W, Pinto G. 2016. Reporte de un caso de artritis-encefalitis caprina en la provincia de San Luis. Boletín no 3 PNSA. Ed. INTA.

Sager RL y Rossanigo CE. 2002. Valores séricos de calcio, fósforo, magnesio, cobre y zinc en cabras de centro-oeste de la Argentina. XIVạ Reunión Científico Técnica de la Asoc. Arg. de Veterinarios de Laboratorios de Diagnóstico (AAVLD), Villa Gral. Belgrano, Córdoba.

Suarez VH. 1997. Diagnóstico de las parasitosis internas de los rumiantes en la región de invernada. Técnicas e Interpretación. Bol. Divulgación Técnica (INTA-Anguil) $56,50 \mathrm{pp}$.

Suarez VH, Micheloud JF, Bertoni EA, Martínez GM. 2013. Caso grave de trichuriasis en cabritos de tambo. Vet. Arg. 30 (304): 1-8.

Suárez VH, Martínez GM, Gianre V, Calvinho L, Rachoski A, Chavez M, Salatin A, Orozco S, Sanchez V, Bertoni, EA. 2014. Relaciones entre el recuento de células somáticas, test de mastitis California conductividad eléctrica y el diagnóstico de mastitis subclínicas en cabras lecheras. Revista de Investigaciones Agropecuarias (INTA) 40: 145-153.

Suárez VH, Rosetto $C B$, Gaido $A B$, Salatin $A O$, Bertoni $E A$, Dodero $A M$, VIñabal $A E$, Pinto $G$, Brihuega $B F$, Romera SA, Maidana S. 2015. Prácticas de manejo y presencia de enfermedades en majadas caprinas de la región del chaco salteño. Vet. Arg. 32(332): 1-24.

Suárez VH, Dodero AM, Nievas JD, Martínez GM, Bertoni $E A$, Salatin $A O$, VIñabal $A E$, Grossberger $G$, Brihuega B, Romera SA, Pinto G. 2016. Presencia de enfermedades en majadas caprinas de las quebradas áridas de Jujuy y Salta. Vet. Arg. 33 (342): 1-25.

Suárez VH, Martínez GM, Nievas JD, Quiroga Roger J. 2017a. Prácticas de manejo y producción en sistemas familiares de cría caprina en las quebradas áridas de Jujuy y Salta. Revista de Investigaciones Agropecuarias (INTA) 43: 186-194. 
Suarez VH, Dodero AM, Almudevar FM, Bertoni EA, Salatin AO, Viñabal AE, Saldaño R, Martínez GM, Micheloud JF, Fiorentino MA, Brihuega BF, Romera SA. 2017b. Presencia de enfermedades y prácticas de manejo en majadas caprinas de los valles templados del noroeste Argentina. Vet. Arg 34(356): 1-25.

Suarez VH, Martínez GM, Viñabal AE, Alfaro JR. 2017c. Epidemiology and effect of gastrointestinal nematodes on dairy goats in Argentina. Onderstepoort J. Vet. Res. 84: a1240.

Suarez VH, Echazú F, Quiroga Roger JA, Viñabal AE. 2018. Parásitos internos de caprinos y ovinos en las regiones de quebradas áridas y la puna de Jujuy (Argentina). Rev. Med. Vet. (B. Aires) 99: 112-116

Suárez VH, Martínez GM, Olmos LH, Arapa C. 2020. Prácticas productivas de los sistemas familiares de cría caprina en los Valles Calchaquies (Payogasta, Salta). FAVE Sección Ciencias Agrarias 19: 7-20.

Thursfield M. 1997. Veterinary Epidemiology. Editorial Acribia S.A., Zaragoza. 339 pp.

Trezeguet M.A, Debenedetti RT, Suárez MF, Barral LE, Ramos M. 2010. Detección de la Artritis-Encefalitis Caprina, en majadas generales, en Argentina. Vet. Arg. 27(270): 1-9.

Viñabal $A E$, Cafrune $M M$, Aguirre $D H$, Bassanetti $A F$, Bertoni EA, Suarez VH. 2015. Propuesta y evaluación de una técnica de sedimentación y tinción con Azul de Metileno (y de una variante) para el diagnóstico de Fasciola hepatica. Vet. Arg. 32 (327): 1-11. 